\title{
Divisor problems of 4 and 3 dimensions
}

\author{
by \\ HoNG-QUAN LiU (Harbin)
}

Introduction. For a positive integer $n$, let the divisor functions $d(4,5,6,7 ; n), d(1,1,2,2 ; n)$ and $d(1,1,2 ; n)$ be defined as in [3], [4]. In this paper we will sharpen our former arguments by proving the following new results regarding the errors of distribution of these divisor functions. We have ( $\varepsilon$ and $x$ are as usual):

Theorem 1.

$$
\sum_{n \leq x} d(4,5,6,7 ; n)=\text { main terms }+O\left(x^{87 / 869+\varepsilon}\right) .
$$

\section{THEOREM 2.}

$$
\sum_{n \leq x} d(1,1,2,2 ; n)=\text { main terms }+O\left(x^{7 / 19+\varepsilon}\right) .
$$

TheOREM 3.

$$
\sum_{n \leq x} d(1,1,2 ; n)=\text { main terms }+O\left(x^{29 / 80+\varepsilon}\right) .
$$

Let $Q_{4}(x)$ be the number of 4-full numbers not exceeding $x$, let $\tau(G)$ be the number of direct factors of a finite Abelian group $G$, and $t(G)$ be the number of unitary factors of $G$, and $T(x)=\sum \tau(G), T^{*}(x)=\sum t(G)$, where the summations are over all $G$ of order not exceeding $x$. Then, as in [3], [4], we have

Corollary 1. $Q_{4}(x)=$ main terms $+O\left(x^{87 / 869+2 \varepsilon}\right)$.

Corollary 2. $T(x)=$ main terms $+O\left(x^{7 / 19+2 \varepsilon}\right)$.

Corollary $3 . T^{*}(x)=$ main terms $+O\left(x^{29 / 80+2 \varepsilon}\right)$.

Note that $87 / 869=0.1001150 \ldots$, which improves the corresponding exponent $6 / 59=0.10169 \ldots$ established in Theorem 2 of [3], and $7 / 19=$ $0.3684 \ldots, 29 / 80=0.3625$ improve respectively the exponents 0.4 and $77 / 208=0.3701 \ldots$ given by Theorems 2 and 1 of [4]. 
In demonstrating these theorems, Theorem 3 of [1] will again play an important role. We will also need to combine other tools existing in papers [2] to [5] of the author. Needless to say, many tedious and elementary calculations will emerge in our treatment, which is inherent in such divisor problems. We will do our best to avoid redundancy.

1. Proof of Theorem 1. We recall a useful lemma (Theorem 3 of [1]).

Lemma 1.1. Let $H \geq 1, X \geq 1, Y \geq 1000$; let $\alpha, \beta$ and $\gamma$ be real numbers with $\alpha \gamma(\gamma-1)(\beta-1) \neq 0$, and let $A>C(\alpha, \beta, \gamma)>0$ and $f(h, x, y)=$ $A h^{\alpha} x^{\beta} y^{\gamma}$. Define

$$
S(H, X, Y)=\sum_{(h, x, y) \in D} C_{1}(h, x) C_{2}(y) e(f(h, x, y)),
$$

where $D$ is a region contained in the rectangle $\{(h, x, y) \mid h \sim H, x \sim X$, $y \sim Y\}$ such that for any fixed pair $\left(h_{0}, x_{0}\right)$, the intersection $D \cap\left\{\left(h_{0}, x_{0}, y\right) \mid\right.$ $y \sim Y\}$ has at most $O(1)$ segments. Also, suppose that $\left|C_{1}(h, x)\right| \leq 1,\left|C_{2}(y)\right|$ $\leq 1$ and $F=A H^{\alpha} X^{\beta} Y^{\gamma} \gg Y$. Then, for $L=\ln ((A+1) H X Y+2)$ and $M=\max \left(1, F Y^{-2}\right)$,

$$
\begin{aligned}
L^{-3} S(H, X, Y) \ll & \sqrt[22]{(H X)^{19} Y^{13} F^{3}}+H X Y^{5 / 8}\left(1+Y^{7} F^{-4}\right)^{1 / 16} \\
& +\sqrt[32]{(H X)^{29} Y^{28} F^{-2} M^{5}}+\sqrt[4]{(H X)^{3} Y^{4} M}
\end{aligned}
$$

We stress that the condition $F \gg Y$ is needed in the proof of this lemma.

We adopt the notations introduced in [3]. In particular, from (7) of [3], we have the following estimate:

$$
\begin{aligned}
\Phi(H ; \boldsymbol{N}) \ll & H^{-1}\left(N_{3}^{2} H^{-1} G^{-1}\right)^{1 / 2} \sum_{h \sim H}\left|\sum_{5} g_{1}\left(n_{1}\right) g_{2}\left(n_{2}\right) g_{3}(u) e(g)\right| \\
& +N_{1}(H G)^{1 / 2} \ln x+x^{13 / 132} .
\end{aligned}
$$

From (23) to (31) of [3], and the estimates on p. 175 there $(\eta=\varepsilon / 8)$,

$$
\begin{aligned}
x^{-\eta} S(a, b, c, d ; \boldsymbol{N}) \ll & \sqrt[33]{x^{3} N_{1}^{12} N_{2}^{2}}+\sqrt[888]{x^{73} N_{1}^{377} N_{2}^{162}} \\
& +\sqrt[76]{x^{6} N_{1}^{34} N_{2}^{19}}+\sqrt[289]{x^{24} N_{1}^{121} N_{2}^{51}}+x^{0.1} .
\end{aligned}
$$

We need two more estimates for $S(a, b, c, d ; \boldsymbol{N})$. First we employ Lemma 1.1 to the triple summation over $n_{1}, n_{2}$ and $u$ in (1.1), with the choice $(h, x, y)$ $=\left(n_{1}, n_{2}, u\right)$. Note that $U \cong H G / N_{3}$; this yields

$$
\begin{aligned}
x^{-\eta} \Phi(H ; \boldsymbol{N}) \ll & \sqrt[22]{(H G)^{5} N_{1}^{19} N_{2}^{19} N_{3}^{9}}+\sqrt[8]{H G\left(N_{1} N_{2}\right)^{8} N_{3}^{3}} \\
& +\sqrt[16]{(H G)^{5}\left(N_{1} N_{2}\right)^{16} N_{3}^{-1}}+\sqrt[32]{(H G)^{10}\left(N_{1} N_{2}\right)^{29} N_{3}^{4}} \\
& +\sqrt[32]{(H G)^{5}\left(N_{1} N_{2}\right)^{29} N_{3}^{14}}+\sqrt[4]{(H G)^{2} N_{1}^{3} N_{2}^{3}} \\
& +\sqrt[4]{H G\left(N_{1} N_{2}\right)^{3} N_{3}^{2}}+x^{0.1} .
\end{aligned}
$$


We put the above estimate in (1) of [3] and choose the parameter $K$ optimally via a well-known lemma (cf. Lemma 3 of [3]) to get

$$
\begin{aligned}
x^{-2 \eta} S(a, b, c, d ; N) \ll & \sqrt[27]{G^{5}\left(N_{1} N_{2}\right)^{24} N_{3}^{14}}+\sqrt[9]{G\left(N_{1} N_{2}\right)^{9} N_{3}^{4}} \\
& +\sqrt[21]{G^{5}\left(N_{1} N_{2}\right)^{21} N_{3}^{4}}+\sqrt[42]{G^{10}\left(N_{1} N_{2}\right)^{39} N_{3}^{14}} \\
& +\sqrt[37]{G^{5}\left(N_{1} N_{2}\right)^{34} N_{3}^{19}}+\sqrt[5]{G N_{1}^{4} N_{2}^{4} N_{3}^{3}} \\
& +\sqrt[6]{G^{2} N_{1}^{5} N_{2}^{5} N_{3}^{2}}+x^{0.1} \\
\ll & \sqrt[108]{x^{5} N_{1}^{61} N_{2}^{66} N_{3}^{31}}+\sqrt[36]{x N_{1}^{29} N_{2}^{30} N_{3}^{11}} \\
& +\sqrt[84]{x^{5} N_{1}^{43} N_{2}^{48} N_{3}^{3}}+\sqrt[148]{x^{5} N_{1}^{101} N_{2}^{106} N_{3}^{51}} \\
& +\sqrt[12]{x N_{1}^{3} N_{2}^{4} N_{3}^{-1}}+\sqrt[20]{x N_{1}^{9} N_{2}^{10} N_{3}^{7}}+x^{0.1} .
\end{aligned}
$$

To pass from (1.3) to (1.4) we have invoked (18) of [3]. By (21) of [3],

$$
x^{-\eta} S(a, b, c, d ; \boldsymbol{N}) \ll \sqrt[8]{x N_{1}^{-3} N_{2}^{-2} N_{3}^{-1}}+x^{0.1} .
$$

From (1.4) and (1.5) we infer that

$$
x^{-2 \eta} S(a, b, c, d ; \boldsymbol{N}) \ll \sum_{1 \leq i \leq 6} P_{i}+x^{0.1},
$$

where

$$
\begin{aligned}
P_{1} & =\min \left(\sqrt[108]{x^{5} N_{1}^{61} N_{2}^{66} N_{3}^{31}}, \sqrt[8]{x N_{1}^{-3} N_{2}^{-2} N_{3}^{-1}}\right) \ll \sqrt[89]{x^{9} N_{1}^{-8} N_{2}}, \\
P_{2} & =\min \left(\sqrt[36]{x N_{1}^{29} N_{2}^{30} N_{3}^{11}}, \sqrt[8]{x N_{1}^{-3} N_{2}^{-2} N_{3}^{-1}}\right) \ll \sqrt[31]{x^{3} N_{1}^{-1} N_{2}^{2}}, \\
P_{3} & =\min \left(\sqrt[84]{x^{5} N_{1}^{43} N_{2}^{48} N_{3}^{3}}, \sqrt[8]{x N_{1}^{-3} N_{2}^{-2} N_{3}^{-1}}\right) \leq \sqrt[54]{x^{4} N_{1}^{17} N_{2}^{21}}, \\
P_{4} & =\min \left(\sqrt[148]{x^{5} N_{1}^{101} N_{2}^{106} N_{3}^{51}}, \sqrt[8]{x N_{1}^{-3} N_{2}^{-2} N_{3}^{-1}}\right) \\
& \leq \sqrt[139]{x^{14} N_{1}^{-13} N_{2}}, \\
P_{5} & =\min \left(\sqrt[12]{x N_{1}^{3} N_{2}^{3}}, \sqrt[8]{x N_{1}^{-3} N_{2}^{-3}}\right) \ll x^{0.1} \\
P_{6} & =\min \left(\sqrt[20]{x N_{1}^{9} N_{2}^{10} N_{3}^{7}}, \sqrt[8]{x N_{1}^{-3} N_{2}^{-2} N_{3}^{-1}}\right) \ll \sqrt[19]{x^{2} N_{1}^{-3} N_{2}^{-1}} .
\end{aligned}
$$

Next, we again apply Lemma 1.1 to the triple summation over $n_{1}, n_{2}$ and $u$ in (1.1), but with the choice $(h, x, y)=\left(n_{1}, u, n_{2}\right)$. This gives

$$
\begin{aligned}
x^{-\eta} \Phi(H ; \boldsymbol{N}) \ll & \sqrt[22]{(H G)^{11} N_{1}^{19} N_{2}^{13} N_{3}^{3}}+(H G)^{1 / 2} N_{1} N_{2}^{5 / 8} \\
& +(H G)^{1 / 4} N_{1} N_{2}^{17 / 16}+\sqrt[32]{(H G)^{11} N_{1}^{29} N_{2}^{28} N_{3}^{3}} \\
& +\sqrt[32]{(H G)^{16} N_{1}^{29} N_{2}^{18} N_{3}^{3}}+\sqrt[4]{H G N_{1}^{3} N_{2}^{4} N_{3}} \\
& +\sqrt[4]{(H G)^{2} N_{1}^{3} N_{2}^{2} N_{3}}+x^{0.1} .
\end{aligned}
$$

We put the above estimate in (1) of [3] and choose $K$ optimally to get 


$$
\begin{aligned}
x^{-2 \eta} S(a, b, c, d ; \boldsymbol{N}) \ll & \sqrt[33]{G^{11} N_{1}^{30} N_{2}^{24} N_{3}^{14}}+\sqrt[12]{G^{4} N_{1}^{12} N_{2}^{9} N_{3}^{4}} \\
& +\sqrt[20]{G^{4} N_{1}^{20} N_{2}^{21} N_{3}^{4}}+\sqrt[43]{G^{11} N_{1}^{40} N_{2}^{39} N_{3}^{14}} \\
& +\sqrt[48]{G^{16} N_{1}^{45} N_{2}^{34} N_{3}^{19}}+\sqrt[5]{G N_{1}^{4} N_{2}^{5} N_{3}^{2}} \\
& +\sqrt[6]{G^{2} N_{1}^{5} N_{2}^{4} N_{3}^{3}}+x \cdot x^{0.1} \\
\ll & \sqrt[132]{x^{11} N_{1}^{43} N_{2}^{30} N_{3}}+\sqrt[12]{x N_{1}^{5} N_{2}^{2}} \\
& +\sqrt[20]{x N_{1}^{13} N_{2}^{14}}+\sqrt[172]{x^{11} N_{1}^{83} N_{2}^{90} N_{3}} \\
& +\sqrt[48]{x^{4} N_{1}^{17} N_{2}^{9}}+\sqrt[20]{x N_{1}^{9} N_{2}^{14} N_{3}^{3}} \\
& +\sqrt[12]{x N_{1}^{3} N_{2}^{2} N_{3}}+x^{0.1}
\end{aligned}
$$

From (1.5) and (1.13) we get

$$
\begin{aligned}
x^{-2 \eta} S(a, b, c, d ; \boldsymbol{N}) \ll & x^{0.1}+\sum_{1 \leq i \leq 4} Q_{i}+\sqrt[12]{x N_{1}^{5} N_{2}^{2}} \\
& +\sqrt[20]{x N_{1}^{13} N_{2}^{14}}+\sqrt[48]{x^{4} N_{1}^{17} N_{2}^{9}}
\end{aligned}
$$

where

$$
\begin{aligned}
Q_{1} & =\min \left(\sqrt[132]{x^{11} N_{1}^{43} N_{2}^{30} N_{3}}, \sqrt[8]{x N_{1}^{-3} N_{2}^{-2} N_{3}^{-1}}\right) \\
& \leq \sqrt[35]{x^{3} N_{1}^{10} N_{2}^{7}}, \\
Q_{2} & =\min \left(\sqrt[172]{x^{11} N_{1}^{83} N_{2}^{90} N_{3}}, \sqrt[8]{x N_{1}^{-3} N_{2}^{-2} N_{3}^{-1}}\right) \\
& \leq \sqrt[45]{x^{3} N_{1}^{20} N_{2}^{22}}, \\
Q_{3} & =\min \left(\sqrt[20]{x N_{1}^{9} N_{2}^{14} N_{3}^{3}}, \sqrt[8]{x N_{1}^{-3} N_{2}^{-2} N_{3}^{-1}}\right) \leq \sqrt[11]{x N_{2}^{2}}, \\
Q_{4} & =\min \left(\sqrt[12]{x N_{1}^{3} N_{2}^{2} N_{3}}, \sqrt[8]{x N_{1}^{-3} N_{2}^{-2} N_{3}^{-1}}\right) \leq x^{0.1}
\end{aligned}
$$

From (31) of [3] we have

$$
x^{-\eta} S(a, b, c, d ; \boldsymbol{N}) \ll \sqrt[28]{\left(x\left(N_{1} N_{2}\right)^{-1}\right)^{3}}+x^{0.1} .
$$

By (1.2) and (1.19) we have

$$
x^{-\eta} S(a, b, c, d ; \boldsymbol{N}) \ll \sum_{1 \leq i \leq 4} R_{i}+x^{0.1},
$$

where

$$
\begin{aligned}
& R_{1}=\min \left(\sqrt[33]{x^{3} N_{1}^{12} N_{2}^{2}}, \sqrt[28]{\left(x\left(N_{1} N_{2}\right)^{-1}\right)^{3}}\right) \leq \sqrt[31]{x^{3} N_{1}^{6}}, \\
& R_{2}=\min \left(\sqrt[888]{x^{73} N_{1}^{377} N_{2}^{162}}, \sqrt[28]{\left(x\left(N_{1} N_{2}\right)^{-1}\right)^{3}}\right) \leq \sqrt[480]{x^{47} N_{1}^{43}}, \\
& R_{3}=\min \left(\sqrt[76]{x^{6} N_{1}^{34} N_{2}^{19}}, \sqrt[28]{\left(x\left(N_{1} N_{2}\right)^{-1}\right)^{3}}\right) \leq \sqrt[152]{x^{15} N_{1}^{9}}, \\
& R_{4}=\min \left(\sqrt[289]{x^{24} N_{1}^{121} N_{2}^{51}}, \sqrt[28]{\left(x\left(N_{1} N_{2}\right)^{-1}\right)^{3}}\right) \leq \sqrt[153]{x^{15} N_{1}^{14}} .
\end{aligned}
$$


From (1.20) to (1.24), we find that the required estimate follows if $N_{1} \leq$ $x^{15 / 869}$. We assume hereafter that $N_{1}>x^{15 / 869}$. From (1.19) and (1.6) to (1.12) we have

$$
x^{-2 \eta} S(a, b, c, d ; \boldsymbol{N}) \ll \sqrt[19]{x^{2} N_{1}^{-3} N_{2}^{-1}}+\sum_{1 \leq i \leq 4} S_{i}+x^{0.1},
$$

where

$$
\begin{aligned}
S_{1} & =\min \left(\sqrt[89]{x^{9} N_{1}^{-8} N_{2}}, \sqrt[28]{\left(x\left(N_{1} N_{2}\right)^{-1}\right)^{3}}\right) \\
& \leq \sqrt[295]{x^{30} N_{1}^{-27}}<x^{87 / 869} \\
S_{2} & =\min \left(\sqrt[31]{x^{3} N_{1}^{-1} N_{2}^{2}}, \sqrt[28]{\left(x\left(N_{1} N_{2}\right)^{-1}\right)^{3}}\right) \\
& \leq \sqrt[149]{x^{15} N_{1}^{-9}} \leq x^{0.1} \\
S_{3} & =\min \left(\sqrt[54]{x^{4} N_{1}^{17} N_{2}^{21}}, \sqrt[28]{\left(x\left(N_{1} N_{2}\right)^{-1}\right)^{3}}\right) \\
& \leq \sqrt[250]{x^{25} N_{1}^{-4}} \leq x^{0.1}, \\
S_{4} & =\min \left(\sqrt[139]{x^{14} N_{1}^{-13} N_{2}}, \sqrt[28]{\left(x\left(N_{1} N_{2}\right)^{-1}\right)^{3}}\right) \\
& \leq \sqrt[445]{x^{45} N_{1}^{-42}} \leq x^{0.1}
\end{aligned}
$$

From (1.25) to (1.29) we have

$$
x^{-2 \eta} S(a, b, c, d ; \boldsymbol{N}) \ll \sqrt[19]{x^{2} N_{1}^{-3} N_{2}^{-1}}+x^{87 / 869} .
$$

By (1.30) and (1.14) to (1.18) we have

$$
x^{-2 \eta} S(a, b, c, d ; \boldsymbol{N}) \ll \sum_{1 \leq i \leq 6} T_{i}+x^{87 / 869},
$$

where

$$
\begin{aligned}
T_{1} & =\min \left(\sqrt[19]{x^{2} N_{1}^{-3} N_{2}^{-1}}, \sqrt[35]{x^{3} N_{1}^{10} N_{2}^{7}}\right) \\
& \leq\left(x^{17} N_{1}^{-11}\right)^{1 / 168} \leq x \cdot 10007 \\
T_{2} & =\min \left(\sqrt[19]{x^{2} N_{1}^{-3} N_{2}^{-1}}, \sqrt[45]{x^{3} N_{1}^{20} N_{2}^{22}}\right) \\
& \leq\left(x^{47} N_{1}^{-46}\right)^{1 / 463} \leq x^{0.1} \\
T_{3} & =\min \left(\sqrt[19]{x^{2} N_{1}^{-3} N_{2}^{-1}}, \sqrt[11]{x N_{2}^{2}}\right) \leq\left(x^{5} N_{1}^{-6}\right)^{1 / 49} \leq x^{0.1}, \\
T_{4} & =\min \left(\sqrt[19]{x^{2} N_{1}^{-3} N_{2}^{-1}}, \sqrt[12]{x N_{1}^{5} N_{2}^{2}}\right) \leq\left(x^{5} N_{1}^{-1}\right)^{1 / 50} \leq x^{0.1}, \\
T_{5} & =\min \left(\sqrt[19]{x^{2} N_{1}^{-3} N_{2}^{-1}}, \sqrt[20]{x N_{1}^{13} N_{2}^{14}}\right) \\
& \leq\left(x^{29} N_{1}^{-29}\right)^{1 / 286} \leq x \sqrt{0.1} \\
T_{6} & =\min \left(\sqrt[19]{x^{2} N_{1}^{-3} N_{2}^{-1}}, \sqrt[48]{x^{4} N_{1}^{17} N_{2}^{9}}\right) \\
& \leq\left(x^{22} N_{1}^{-10}\right)^{1 / 219} \leq x^{0.1} .
\end{aligned}
$$

By (1.30) to (1.37), we have completed the proof. 
2. Proof of Theorem 2. Let $(a, b, c, d)$ be any permutation of $(1,1,2,2)$. It suffices to obtain

$$
S(a, b, c, d ; \boldsymbol{N}) \ll x^{7 / 19+4 \eta},
$$

where $\eta=\varepsilon / 8, \boldsymbol{N}=\left(N_{1}, N_{2}, N_{3}\right), N_{1}, N_{2}$ and $N_{3}$ are positive integers with

$$
N_{1} \ll N_{2} \ll N_{3}, \quad N_{1}^{a} N_{2}^{b} N_{3}^{c+d} \ll x, \quad N_{1} N_{2} N_{3}>x^{1 / 3},
$$

and the sum $S(a, b, c, d ; \boldsymbol{N})$ is defined on p. 199 of [4]. We will retain many familiar notations used in both [3] and [4].

The case of $(a, b, c, d)=(1,1,2,2)$ can be dealt with immediately. In fact, from (2.2) we have $N_{1} N_{2} \ll x^{1 / 3}$, thus $\left(G N_{1} N_{2} N_{3}\right)^{1 / 2} \ll\left(x N_{1} N_{2}\right)^{1 / 4} \ll$ $x^{1 / 3}$, and the required estimate follows from Lemma 6 of [4].

For $(a, b, c, d)=(1,2,1,2)$, by $(2.2)$ we have $N_{1}^{3} N_{3}^{3} \ll N_{1} N_{2}^{2} N_{3}^{3} \ll x$, thus again $\left(G N_{1} N_{2} N_{3}\right)^{1 / 2} \ll x^{1 / 3}$, and the required estimate follows.

We now show

$$
S(2,1,1,2 ; \boldsymbol{N}) \ll x^{4 / 11+\varepsilon} .
$$

To this end, we first proceed similarly to pp. 167-170 of [3]. This yields, similarly to (7) of [3],

$$
\begin{gathered}
\Phi(H ; \boldsymbol{N}) \ll H^{-1}\left(N_{3}^{2} H^{-1} G^{-1}\right)^{1 / 2} \sum_{h \sim H}\left|\sum_{1} F\left(n_{1}\right) R\left(n_{2}\right) S(u) e(g)\right| \\
+N_{1}(H G)^{1 / 2} \ln x+x^{13 / 36}
\end{gathered}
$$

(for an explanation of the error term $x^{13 / 36}$, cf. p. 199 of [4]), where $\sum_{1}$ means summation over $n_{1}, n_{2}$ and $u$ with

$$
1<n_{1}<n_{2}, \quad N_{v} \leq n_{v}<2 N_{v}(v=1,2), \quad U_{1}<u<U_{2},
$$

and $G, U_{1}, U_{2}$ and the function $g$ are defined on p. 169 of [3]. In particular, $g=C_{2}\left(x n_{1}^{-2} n_{2}^{-1} h^{2} u\right)^{1 / 3}$. Moreover, $F(\cdot), R(\cdot), S(\cdot)$ are suitable monomials with absolute values $\cong 1$. We can apply Lemma 1 of [3] one more time, to the variable $n_{2}$ of (2.4). We have

$$
\begin{aligned}
\Phi(H ; \boldsymbol{N}) \ll & N_{2} N_{3}\left(H^{2} G\right)^{-1} \\
& \times \sum_{h \sim H} \sum_{n_{1} \sim N_{1}}\left|\sum_{U_{1}<u<U_{2}} \sum_{V_{1}<v<V_{2}} T(u) Q(v) e\left(g_{1}\right)\right| \\
& +N_{1}(H G)^{1 / 2} \ln x+x^{13 / 36},
\end{aligned}
$$

where $V_{i}=V_{i}\left(h, n_{1}, u\right)(i=1,2),|T(u)| \leq 1,|Q(v)| \leq 1$, and $g_{1}=$ $C_{3}\left(h^{2} x_{u v n_{1}^{-2}}\right)^{1 / 4}$. We can relax the condition $U_{1}<u<U_{2}$ to $u \cong U:=$ $H G N_{3}^{-1}$ and the condition $V_{1}<v<V_{2}$ to $v \cong V:=H G N_{2}^{-1}$ consecutively by means of Lemma 5 of [3] (note that we can assume that $x$ is quadratic irrational, cf. p. 168 of [3]); we thus deduce from (2.5) that 


$$
\begin{aligned}
x^{-\eta} \Phi(H ; \boldsymbol{N}) \ll & N_{2} N_{3}\left(H^{2} G\right)^{-1} \\
& \times \sum_{h \sim H} \sum_{n_{1} \sim N_{1}}\left|\sum_{u \cong U} \sum_{v \cong V} T_{1}(u) Q_{1}(v) e\left(g_{1}\right)\right| \\
& +N_{1}(H G)^{1 / 2}+x^{13 / 36},
\end{aligned}
$$

where $\left|T_{1}(u)\right|,\left|Q_{1}(v)\right| \leq 1$. From (2.6) it is evident that

$$
\begin{aligned}
x^{-2 \eta} \Phi(H ; \boldsymbol{N}) \ll & N_{2} N_{3}\left(H^{2} G\right)^{-1} \\
& \times \sum_{h \sim H} \sum_{n_{1} \sim N_{1}}\left|\sum_{w \cong W} K(w) e\left(C_{3}\left(h^{2} x w n_{1}^{-2}\right)^{1 / 4}\right)\right| \\
& +N_{1}(H G)^{1 / 2}+x^{13 / 36},
\end{aligned}
$$

where $W=U V=(H G)^{2} N_{2}^{-1} N_{3}^{-1}$ and $|K(w)| \leq 1$.

If $H G \ll N_{2} N_{3}$, we apply Lemma 1.1 to the triple exponential sum in $(2.7)$, with $(h, x, y)=\left(h, n_{1}, w\right)$, to get

$$
\begin{aligned}
x^{-3 \eta} \Phi(H ; N) \ll & \sqrt[22]{H^{4} G^{7} N_{1}^{19}\left(N_{2} N_{3}\right)^{9}}+(H G)^{1 / 4} N_{1}\left(N_{2} N_{3}\right)^{3 / 8} \\
& +(H G)^{7 / 8} N_{1}\left(N_{2} N_{3}\right)^{-1 / 16} \\
& +\sqrt[32]{H^{19} G^{22} N_{1}^{29}\left(N_{2} N_{3}\right)^{4}} \\
& +\sqrt[32]{H^{4} G^{4} N_{1}^{29}\left(N_{2} N_{3}\right)^{14}}+\sqrt[4]{H^{3} G^{4} N_{1}^{3}} \\
& +\sqrt[4]{G N_{1}^{3}\left(N_{2} N_{3}\right)^{2}}+x^{13 / 36} \\
\ll & \sqrt[22]{G^{3} N_{1}^{19}\left(N_{2} N_{3}\right)^{13}}+N_{1}\left(N_{2} N_{3}\right)^{5 / 8} \\
& +\sqrt[32]{G^{3} N_{1}^{29}\left(N_{2} N_{3}\right)^{18}}+\sqrt[4]{G N_{1}^{3}\left(N_{2} N_{3}\right)^{2}} \\
& +N_{1}(H G)^{13 / 16}+\sqrt[32]{H^{19} G^{22} N_{1}^{29}\left(N_{2} N_{3}\right)^{4}} \\
& +\sqrt[4]{H^{3} G^{4} N_{1}^{3}}+x^{13 / 36}
\end{aligned}
$$

If $N_{1} \geq x^{1 / 22}$, we have $\left(G N_{1} N_{2} N_{3}\right)^{1 / 2}=\left(x N_{2} N_{3}\right)^{1 / 4} \ll\left(x^{3} N_{1}^{-2}\right)^{1 / 8} \ll$ $x^{4 / 11}$, and (2.3) follows from Lemma 6 of [4]. We now assume that $N_{1}<$ $x^{1 / 22}$. Then we easily see that the total contribution of the first four terms in $(2.8)$ is $\ll x^{0.34}$. In fact, since $N_{1} N_{2} N_{3} \ll x^{1 / 2}$,

$$
\begin{aligned}
\sqrt[22]{\left.G^{3} N_{1}^{19}\left(N_{2} N_{3}\right)\right)^{13}} & \ll \sqrt[44]{x^{3} N_{1}^{32}\left(N_{2} N_{3}\right)^{23}} \ll \sqrt[88]{x^{29} N_{1}^{18}} \ll x^{0.34}, \\
N_{1}\left(N_{2} N_{3}\right)^{5 / 8} \ll \sqrt[16]{x^{5} N_{1}^{6}} \ll x^{0.33} & \\
\sqrt[32]{G^{3} N_{1}^{29}\left(N_{2} N_{3}\right)^{18}} & \ll \sqrt[64]{x^{3} N_{1}^{52}\left(N_{2} N_{3}\right)^{33}} \ll \sqrt[128]{x^{39} N_{1}^{38}} \ll x^{0.32}, \\
\sqrt[4]{G N_{1}^{3}\left(N_{2} N_{3}\right)^{2}} & \ll \sqrt[8]{x N_{1}^{4}\left(N_{2} N_{3}\right)^{3}} \ll \sqrt[16]{x^{5} N_{1}^{2}} \ll x^{0.32}
\end{aligned}
$$

Thus from (2.8) we get

$$
\begin{aligned}
x^{-3 \eta} \Phi(H ; \boldsymbol{N}) \ll & N_{1}(H G)^{13 / 16}+\sqrt[32]{H^{19} G^{22} N_{1}^{29}\left(N_{2} N_{3}\right)^{4}} \\
& +\sqrt[4]{H^{3} G^{4} N_{1}^{3}}+x^{13 / 36} .
\end{aligned}
$$


If $H G \gg N_{2} N_{3}$, we go back to the original definition for $\Phi(H ; \boldsymbol{N})$, and we produce a new integral variable $q$ from $n_{2}$ and $n_{3}$ such that $q=n_{2} n_{3}$. Since $H G \gg N_{2} N_{3}$, Lemma 1.1 is applicable with $(h, x, y)=\left(h, n_{1}, q\right)$, and we get

$$
\begin{aligned}
x^{-\eta} \Phi(H ; \boldsymbol{N}) \ll & \sqrt[22]{G^{3} N_{1}^{19}\left(N_{2} N_{3}\right)^{13}}+N_{1}\left(N_{2} N_{3}\right)^{5 / 8}+N_{1}(H G)^{13 / 16} \\
& +\sqrt[32]{H^{19} G^{22} N_{1}^{29}\left(N_{2} N_{3}\right)^{4}}+\sqrt[32]{G^{3} N_{1}^{29}\left(N_{2} N_{3}\right)^{18}} \\
& +\sqrt[4]{H^{3} G^{4} N_{1}^{3}}+\sqrt[4]{G N_{1}^{3}\left(N_{2} N_{3}\right)^{2}}+x^{13 / 36} \\
\ll & N_{1}(H G)^{13 / 16}+\sqrt[32]{H^{19} G^{22} N_{1}^{29}\left(N_{2} N_{3}\right)^{4}} \\
& +\sqrt[4]{H^{3} G^{4} N_{1}^{3}}+x^{13 / 36} .
\end{aligned}
$$

Thus we see that (2.9) always holds. We put the estimate (2.9) in (1) of [3] and choose the parameter $K$ optimally via Lemma 3 of [3] to get

$$
\begin{aligned}
x^{-4 \eta} S(2,1,1,2 ; \boldsymbol{N}) \ll & \sqrt[29]{G^{13} N_{1}^{29}\left(N_{2} N_{3}\right)^{13}}+\sqrt[51]{G^{22} N_{1}^{48}\left(N_{2} N_{3}\right)^{23}} \\
& +\sqrt[7]{G^{4} N_{1}^{6}\left(N_{2} N_{3}\right)^{3}}+x^{13 / 36} \\
\ll & \sqrt[58]{x^{13} N_{1}^{32}\left(N_{2} N_{3}\right)^{13}}+\sqrt[51]{x^{11} N_{1}^{26}\left(N_{2} N_{3}\right)^{12}} \\
& +\sqrt[7]{x^{2} N_{1}^{2} N_{2} N_{3}}+x^{13 / 36} \\
\ll & \sqrt[116]{x^{39} N_{1}^{38}}+\sqrt[51]{x^{17} N_{1}^{14}} \\
& +\sqrt[14]{x^{5} N_{1}^{2}}+x^{13 / 36} \ll x^{4 / 11},
\end{aligned}
$$

which proves $(2.3)$.

We proceed to estimate $S(2,2,1,1 ; \boldsymbol{N})$; the remaining two cases with $(a, b, c, d)=(2,1,2,1)$ and $(1,2,2,1)$ can be treated similarly. As in (7) of $[3]$, we get

$$
\begin{aligned}
\Phi(H ; \boldsymbol{N}) \ll & H^{-1}\left(N_{3}^{2} H^{-1} G^{-1}\right)^{1 / 2} \\
& \times \sum_{h \sim H}\left|\sum_{2} g_{1}\left(n_{1}\right) g_{2}\left(n_{2}\right) g_{3}(u) e\left(C\left(\frac{x h u}{n_{1}^{2} n_{2}^{2}}\right)^{1 / 2}\right)\right| \\
& +N_{1} N_{2} \ln x+\left(H x\left(N_{1} N_{2} N_{3}\right)^{-1}\right)^{1 / 2}+x^{13 / 36},
\end{aligned}
$$

where $\sum_{2}$ means summation over lattice points $\left(n_{1}, n_{2}, u\right)$ such that

$$
\begin{gathered}
1 \leq n_{1}<n_{2}, \quad n_{1} \sim N_{1}, \quad n_{2} \sim N_{2}, \\
h x\left(n_{1} n_{2} M_{2}\right)^{-2}<u<h x\left(n_{1} n_{2} M_{1}\right)^{-2},
\end{gathered}
$$

and where $M_{1}=\max \left(N_{3}, n_{2}\right), M_{2}=\min \left(\left(x n_{1}^{-2} n_{2}^{-2}\right)^{1 / 2}, 2 N_{3}\right), g_{i}(\cdot)$ are monomials with $\left|g_{i}(\cdot)\right| \cong 1$. By an appeal to Lemma 5 of [3], we can relax the summation range to $u \cong U=H G N_{3}^{-1}$. Then we can produce a double sum in (2.10) by setting $h u=r$ and $n_{1} n_{2}=s$. This yields 


$$
\begin{aligned}
x^{-\eta} \Phi(H ; \boldsymbol{N}) \ll & H^{-1}\left(N_{3}^{2} H^{-1} G^{-1}\right)^{1 / 2} \sum_{r \cong R}\left|\sum_{s \cong S} B(s) e\left(C\left(x r s^{-2}\right)^{1 / 2}\right)\right| \\
& +N_{1} N_{2}+\left(H x\left(N_{1} N_{2} N_{3}\right)^{-1}\right)^{1 / 2}+x^{13 / 36},
\end{aligned}
$$

where $R=H^{2} G N_{3}^{-1}, S=N_{1} N_{2}$, and $|B(s)| \leq 1$.

If $H G \gg N_{1} N_{2}$, then Lemma 1.1 is applicable to the exponential sum in $(2.11)$ with $(h, x, y)=(1, r, s)$, and we get

$$
\begin{aligned}
x^{-2 \eta} \Phi(H ; \boldsymbol{N}) \ll & \sqrt[22]{H^{8} G^{11}\left(N_{1} N_{2}\right)^{13} N_{3}^{3}}+(H G)^{1 / 2}\left(N_{1} N_{2}\right)^{5 / 8} \\
& +\sqrt[16]{(H G)^{4}\left(N_{1} N_{2}\right)^{17}}+\sqrt[32]{H^{8} G^{11}\left(N_{1} N_{2}\right)^{28} N_{3}^{3}} \\
& +\sqrt[32]{H^{13} G^{16}\left(N_{1} N_{2}\right)^{18} N_{3}^{3}}+\sqrt[4]{G\left(N_{1} N_{2}\right)^{4} N_{3}} \\
& +\sqrt[4]{H G^{2}\left(N_{1} N_{2}\right)^{2} N_{3}}+N_{1} N_{2} \\
& +\left(H x\left(N_{1} N_{2} N_{3}\right)^{-1}\right)^{1 / 2}+x^{\theta}
\end{aligned}
$$

$(\theta=13 / 36)$. Secondly, we apply Lemma 1.1 to the exponential sum in (2.10) with $(h, x, y)=\left(h u, n_{1}, n_{2}\right)$ to get

$$
\begin{aligned}
x^{-2 \eta} \Phi(H ; N) \ll & \sqrt[22]{H^{8} G^{11} N_{1}^{19} N_{2}^{13} N_{3}^{3}}+(H G)^{1 / 2} N_{1} N_{2}^{5 / 8} \\
& +(H G)^{1 / 4} N_{1} N_{2}^{17 / 16}+\sqrt[32]{H^{8} G^{11} N_{1}^{29} N_{2}^{28} N_{3}^{3}} \\
& +\sqrt[32]{H^{13} G^{16} N_{1}^{29} N_{2}^{18} N_{3}^{3}}+\sqrt[4]{G N_{1}^{3} N_{2}^{4} N_{3}} \\
& +\sqrt[4]{H G^{2} N_{1}^{3} N_{2}^{2} N_{3}}+N_{1} N_{2} \\
& +\left(H x\left(N_{1} N_{2} N_{3}\right)^{-1}\right)^{1 / 2}+x^{\theta} .
\end{aligned}
$$

If $H G \gg N_{1} N_{2}$ is not true, that is, $H G \ll N_{1} N_{2}$, from (2.13) we get

$$
\begin{aligned}
x^{-2 \eta} \Phi(H ; \boldsymbol{N}) \ll & \sqrt[22]{G^{3} N_{1}^{27} N_{2}^{21} N_{3}^{3}}+N_{1}^{3 / 2} N_{2}^{9 / 8}+N_{1}^{5 / 4} N_{2}^{21 / 16} \\
& +\sqrt[32]{G^{3} N_{1}^{37} N_{2}^{36} N_{3}^{3}}+\sqrt[32]{G^{3} N_{1}^{42} N_{2}^{31} N_{3}^{3}} \\
& +\sqrt[4]{G N_{1}^{3} N_{2}^{4} N_{3}}+\sqrt[4]{G N_{1}^{4} N_{2}^{3} N_{3}} \\
& +N_{1} N_{2}+\left(H x\left(N_{1} N_{2} N_{3}\right)^{-1}\right)^{1 / 2}+x^{\theta} \\
\ll & \sqrt[22]{x^{3} N_{1}^{21} N_{2}^{15}}+\sqrt[8]{N_{1}^{12} N_{2}^{9}}+\sqrt[16]{N_{1}^{20} N_{2}^{21}} \\
& +\sqrt[32]{x^{3} N_{1}^{31} N_{2}^{30}}+\sqrt[32]{x^{3} N_{1}^{36} N_{2}^{25}}+\sqrt[4]{x N_{1} N_{2}^{2}} \\
& +\sqrt[4]{x N_{1}^{2} N_{2}}+N_{1} N_{2}+\left(H x\left(N_{1} N_{2} N_{3}\right)^{-1}\right)^{1 / 2}+x^{\theta} .
\end{aligned}
$$

By Lemma 6 of $[4], S(2,2,1,1 ; \boldsymbol{N}) \ll\left(\left(x\left(N_{1} N_{2}\right)^{-1}\right)^{1 / 2}+x^{\theta}\right) x^{\eta}$, thus the required estimate follows if $N_{1} N_{2}>x^{5 / 19}$. We assume hereafter that $N_{1} N_{2} \leq$ $x^{5 / 19}$. Then from (2.14) we have, using the fact that $N_{1} \ll N_{2}$,

$$
x^{-2 \eta} \Phi(H ; \boldsymbol{N}) \ll \sqrt[4]{x\left(N_{1} N_{2}\right)^{2}}+\left(H x\left(N_{1} N_{2} N_{3}\right)^{-1}\right)^{1 / 2}+x^{\theta} .
$$

Note that (2.15) is derived when $N_{1} N_{2} \gg H G$, thus we find from (2.12) 
that the following estimate always holds:

$$
\begin{aligned}
x^{-2 \eta} \Phi(H ; \boldsymbol{N}) \ll & \sqrt[22]{H^{8} G^{11}\left(N_{1} N_{2}\right)^{13} N_{3}^{3}}+(H G)^{1 / 2}\left(N_{1} N_{2}\right)^{5 / 8} \\
& +\sqrt[16]{(H G)^{4}\left(N_{1} N_{2}\right)^{17}}+\sqrt[32]{H^{8} G^{11}\left(N_{1} N_{2}\right)^{28} N_{3}^{3}} \\
& +\sqrt[32]{H^{13} G^{16}\left(N_{1} N_{2}\right)^{18} N_{3}^{3}}+\sqrt[4]{x\left(N_{1} N_{2}\right)^{2}} \\
+ & \left(H x\left(N_{1} N_{2} N_{3}\right)^{-1}\right)^{1 / 2}+x^{\theta} \\
& +\sqrt[4]{H G^{2}\left(N_{1} N_{2}\right)^{2} N_{3}} \\
=: & E_{1}(H)+\sqrt[4]{H G^{2}\left(N_{1} N_{2}\right)^{2} N_{3}} .
\end{aligned}
$$

We want to diminish the term $\sqrt[4]{H G^{2}\left(N_{1} N_{2}\right)^{2} N_{3}}$ in (2.16). To this end, we first note that if $H<N_{3}$, then Lemma 1.1 is applicable to the exponential sum in (2.11) with $(h, x, y)=(1, s, r)$, and this gives

$$
\begin{aligned}
x^{-2 \eta} \Phi(H ; \boldsymbol{N}) \ll & \sqrt[22]{H^{-4} G^{5}\left(N_{1} N_{2}\right)^{19} N_{3}^{9}}+\sqrt[8]{H^{-2} G\left(N_{1} N_{2}\right)^{8} N_{3}^{3}} \\
& +\sqrt[16]{H^{6} G^{5}\left(N_{1} N_{2}\right)^{16} N_{3}^{-1}} \\
& +\sqrt[32]{H^{6} G^{10}\left(N_{1} N_{2}\right)^{29} N_{3}^{4}} \\
& +\sqrt[32]{H^{-9} G^{5}\left(N_{1} N_{2}\right)^{29} N_{3}^{14}}+\sqrt[4]{H^{2} G^{2}\left(N_{1} N_{2}\right)^{3}} \\
& +\sqrt[4]{H^{-1} G\left(N_{1} N_{2}\right)^{3} N_{3}^{2}}+\left(\frac{H x}{N_{1} N_{2} N_{3}}\right)^{1 / 2}+x^{\theta} \\
=: & E_{2}(H) .
\end{aligned}
$$

If $H \geq N_{3}$, then from (2.16) we get

$$
x^{-2 \eta} \Phi(H ; \boldsymbol{N}) \ll E_{1}(H)+\left(H G N_{1} N_{2}\right)^{1 / 2} .
$$

By (2.17) and (2.18), we always have

$$
x^{-2 \eta} \Phi(H ; \boldsymbol{N}) \ll E_{1}(H)+E_{2}(H)+\left(H G N_{1} N_{2}\right)^{1 / 2} .
$$

From (2.16) and (2.19) we get

$$
\begin{aligned}
x^{-2 \eta} \Phi(H ; \boldsymbol{N}) \ll & E_{1}(H)+\left(H G N_{1} N_{2}\right)^{1 / 2} \\
& +\min \left(E_{2}(H), \sqrt[4]{H G^{2}\left(N_{1} N_{2}\right)^{2} N_{3}}\right),
\end{aligned}
$$

where

$$
\begin{aligned}
\min & \left(E_{2}(H), \sqrt[4]{H G^{2}\left(N_{1} N_{2}\right)^{2} N_{3}}\right) \\
\ll & E_{1}(H)+\sum_{1 \leq i \leq 4} A_{i}+\sqrt[16]{H^{6} G^{5}\left(N_{1} N_{2}\right)^{16} N_{3}^{-1}} \\
& +\sqrt[32]{H^{6} G^{10}\left(N_{1} N_{2}\right)^{29} N_{3}^{4}}+\sqrt[4]{H^{2} G^{2}\left(N_{1} N_{2}\right)^{3}}, \\
A_{1}= & \min \left(\sqrt[22]{H^{-4} G^{5}\left(N_{1} N_{2}\right)^{19} N_{3}^{9}}, \sqrt[4]{H G^{2}\left(N_{1} N_{2}\right)^{2} N_{3}}\right) \\
\leq & \sqrt[38]{G^{13}\left(N_{1} N_{2}\right)^{27} N_{3}^{13}},
\end{aligned}
$$




$$
\begin{aligned}
A_{2} & =\min \left(\sqrt[8]{H^{-2} G\left(N_{1} N_{2}\right)^{8} N_{3}^{3}}, \sqrt[4]{H G^{2}\left(N_{1} N_{2}\right)^{2} N_{3}}\right) \\
& \leq \sqrt[16]{G^{5}\left(N_{1} N_{2}\right)^{12} N_{3}^{5}}, \\
A_{3} & =\min \left(\sqrt[32]{H^{-9} G^{5}\left(N_{1} N_{2}\right)^{29} N_{3}^{14}}, \sqrt[4]{H G^{2} N_{1}^{2} N_{2}^{2} N_{3}}\right) \\
& \leq \sqrt[68]{G^{23}\left(N_{1} N_{2}\right)^{47} N_{3}^{23}}, \\
A_{4} & =\min \left(\sqrt[4]{H^{-1} G\left(N_{1} N_{2}\right)^{3} N_{3}^{2}}, \sqrt[4]{H G^{2} N_{1}^{2} N_{2}^{2} N_{3}}\right) \\
& \leq \sqrt[8]{G^{3} N_{1}^{5} N_{2}^{5} N_{3}^{3}} .
\end{aligned}
$$

Since $G=x\left(N_{1}^{2} N_{2}^{2} N_{3}\right)^{-1}$ and $N_{1} N_{2} \leq x^{5 / 19}$, it is easy to verify that $A_{1}, A_{2}, A_{3} \ll x^{0.35}<x^{\theta}$. If $N_{1} N_{2}<x^{2 / 19}$, by Lemma 2 of [5] with $(k, \lambda)=$ $(1 / 2,1 / 2)$ we get

$$
x^{-\eta} S(2,2,1,1 ; \boldsymbol{N}) \ll N_{1} N_{2}\left(x\left(N_{1} N_{2}\right)^{-2}\right)^{1 / 3} \ll x^{7 / 19} .
$$

We assume hereafter that $N_{1} N_{2} \geq x^{2 / 19}$. Thus $A_{4} \ll x^{\varphi}, \varphi=7 / 19$. From these observations and (2.20) to (2.25), we achieve that

$$
x^{-2 \eta} \Phi(H ; \boldsymbol{N}) \ll E_{1}(H)+E_{3}(H),
$$

where

$$
\begin{aligned}
E_{3}(H)= & \sqrt[16]{H^{6} G^{5}\left(N_{1} N_{2}\right)^{16} N_{3}^{-1}}+\sqrt[32]{H^{6} G^{10}\left(N_{1} N_{2}\right)^{29} N_{3}^{4}} \\
& +\sqrt[4]{H^{2} G^{2} N_{1}^{3} N_{2}^{3}}+x^{\varphi} .
\end{aligned}
$$

We put the estimate of (2.26) in (1) of [3] and choose $K$ optimally via Lemma 3 of [3] to get

$$
\begin{aligned}
x^{-3 \eta} S(2,2,1,1 ; N) \ll & \sqrt[30]{G^{11}\left(N_{1} N_{2}\right)^{21} N_{3}^{11}}+\sqrt[12]{G^{4} N_{1}^{9} N_{2}^{9} N_{3}^{4}} \\
& +\sqrt[20]{G^{4}\left(N_{1} N_{2}\right)^{21} N_{3}^{4}}+\sqrt[40]{G^{11}\left(N_{1} N_{2}\right)^{36} N_{3}^{11}} \\
& +\sqrt[45]{G^{16}\left(N_{1} N_{2}\right)^{31} N_{3}^{16}}+\sqrt[4]{x N_{1}^{2} N_{2}^{2}} \\
& +\sqrt[22]{G^{5}\left(N_{1} N_{2}\right)^{22} N_{3}^{5}}+\sqrt[38]{G^{10}\left(N_{1} N_{2}\right)^{35} N_{3}^{10}} \\
& +\sqrt[6]{G^{2} N_{1}^{5} N_{2}^{5} N_{3}^{2}}+x^{\varphi} \\
\ll & \sqrt[30]{x^{11} J^{-1}}+\sqrt[12]{x^{4} J}+\sqrt[20]{x^{4} J^{13}}+\sqrt[40]{x^{11} J^{14}} \\
& +\sqrt[45]{x^{16} J^{-1}}+\sqrt[4]{x J^{2}}+\sqrt[22]{x^{5} J^{12}} \\
& +\sqrt[38]{x^{10} J^{15}}+\sqrt[6]{x^{2} J}+x^{\varphi} \\
\ll & \sqrt[6]{x^{2} J}+\sqrt[4]{x J^{2}}+\sqrt[20]{x^{4} J^{13}}+\sqrt[22]{x^{5} J^{12}}+x^{\varphi}
\end{aligned}
$$

where, for simplicity, $J:=N_{1} N_{2}$. Suppose

$$
H^{8} G^{5} \geq\left(N_{1} N_{2}\right)^{4} N_{3}^{3} x^{\delta}, \quad \delta=\varepsilon^{2} .
$$

Then we find that Lemma 2.4 of [2] is applicable to the exponential sum of (2.11) with $(x, y)=(s, r)$. This gives 


$$
\begin{aligned}
x^{-2 \eta} \Phi(H ; \boldsymbol{N}) & \ll \sqrt[40]{G^{15} H^{2}\left(N_{1} N_{2}\right)^{29} N_{3}^{13}} \\
& +\sqrt[10]{H^{3} G^{5}\left(N_{1} N_{2}\right)^{6} N_{3}^{2}}+\sqrt[40]{H^{14}\left(N_{1} N_{2}\right)^{33} N_{3} G^{15}} \\
& +\sqrt[10]{G^{5} H^{6}\left(N_{1} N_{2}\right)^{7} N_{3}^{-1}}+\sqrt[4]{H^{3} G^{2} N_{1}^{3} N_{2}^{3} N_{3}^{-1}} \\
& +\sqrt[20]{H^{9} G^{10}\left(N_{1} N_{2}\right)^{13} N_{3}}+\sqrt[4]{H G^{2} N_{1}^{2} N_{2}^{2} N_{3}} \\
& +\sqrt[20]{\left(N_{1} N_{2}\right)^{14} N_{3}^{13}}+\sqrt[20]{\left(N_{1} N_{2}\right)^{16} N_{3}^{7}} \\
& +\sqrt[10]{H^{8} G^{5} N_{1}^{6} N_{2}^{6} N_{3}^{-3}}+\left(H x\left(N_{1} N_{2} N_{3}\right)^{-1}\right)^{1 / 2}+x^{\theta} \\
& =: E_{4}(H) .
\end{aligned}
$$

If (2.28) is not true, that is, if we have $H^{8}<G^{-5} N_{1}^{4} N_{2}^{4} N_{3}^{3} x^{\delta}$, then we use the estimate of (2.13) to get

$$
\begin{aligned}
x^{-3 \eta} \Phi(H ; N) \ll & \sqrt[22]{G^{6} N_{1}^{23} N_{2}^{17} N_{3}^{6}}+\sqrt[16]{G^{3} N_{1}^{20} N_{2}^{14} N_{3}^{3}} \\
& +\sqrt[32]{G^{3} N_{1}^{36} N_{2}^{38} N_{3}^{3}}+\sqrt[32]{G^{6} N_{1}^{33} N_{2}^{32} N_{3}^{6}} \\
& +\sqrt[256]{G^{63} N_{1}^{284} N_{2}^{196} N_{3}^{63}}+\sqrt[4]{G N_{1}^{3} N_{2}^{4} N_{3}} \\
& +\sqrt[32]{G^{11} N_{1}^{28} N_{2}^{20} N_{3}^{11}} \\
& +\left(H x\left(N_{1} N_{2} N_{3}\right)^{-1}\right)^{1 / 2}+x^{\theta} \\
\ll & \sqrt[22]{x^{6} N_{1}^{11} N_{2}^{5}}+\sqrt[16]{x^{3} N_{1}^{14} N_{2}^{8}}+\sqrt[32]{x^{3} N_{1}^{30} N_{2}^{32}} \\
& +\sqrt[32]{x^{6} N_{1}^{21} N_{2}^{20}}+\sqrt[256]{x^{63} N_{1}^{158} N_{2}^{70}} \\
& +\sqrt[32]{x^{11} N_{1}^{6} N_{2}^{-2}}+\sqrt[4]{x N_{1} N_{2}^{2}} \\
& +\left(H x\left(N_{1} N_{2} N_{3}\right)^{-1}\right)^{1 / 2}+x^{\theta} \\
\ll & \sqrt[22]{x^{6} J^{8}}+\sqrt[16]{x^{3} J^{11}}+\sqrt[32]{x^{3} J^{32}} \\
& +\sqrt[32]{x^{6} J^{20.5}}+\sqrt[256]{x^{63} J^{114}} \\
& +\sqrt[32]{x^{11} J^{2}}+\sqrt[4]{x N_{1} N_{2}^{2}} \\
& +\left(H x\left(N_{1} N_{2} N_{3}\right)^{-1}\right)^{1 / 2}+x^{\theta} \\
\ll & \sqrt[4]{x N_{1} N_{2}^{2}}+\left(H x\left(N_{1} N_{2} N_{3}\right)^{-1}\right)^{1 / 2}+x^{\varphi} .
\end{aligned}
$$

To diminish the term $\sqrt[4]{x N_{1} N_{2}^{2}}$ in $(2.30)$ we can treat the double sum over $\left(u, n_{2}\right)$ in (2.10) similarly to those given by (3), (4), (10) of [4] by using Lemma 1.5 of [2], and we thus obtain similarly to (11) of [4] the following estimate:

$$
\begin{aligned}
x^{-\eta} \Phi(H ; \boldsymbol{N}) \ll & N_{1}\left(\sqrt[12]{(H G)^{10} N_{2} N_{3}}+\sqrt[16]{(H G)^{10} N_{2}^{5} N_{3}^{3}}\right. \\
& +\sqrt[4]{(H G)^{3} N_{3}}+\sqrt[80]{(H G)^{58} N_{2}^{29} N_{3}^{-5}} \\
& +\sqrt[64]{(H G)^{54} N_{3}^{-3} N_{2}^{11}}+\sqrt[16]{(H G)^{14} N_{2}}
\end{aligned}
$$




$$
\begin{aligned}
& +\sqrt[128]{(H G)^{114} N_{3}^{3} N_{2}^{5}}+(H G)^{7 / 8} \\
& \left.+\sqrt[64]{(H G)^{58} N_{3}^{3} N_{2}^{-3}}\right) \\
& +\left(H x\left(N_{1} N_{2} N_{3}\right)^{-1}\right)^{1 / 2}+x^{\theta} \\
& \ll \sqrt[48]{G^{15} N_{1}^{68} N_{2}^{24} N_{3}^{19}}+\sqrt[64]{G^{15} N_{1}^{84} N_{2}^{40} N_{3}^{27}} \\
& +\sqrt[32]{G^{9} N_{1}^{44} N_{2}^{12} N_{3}^{17}}+\sqrt[320]{G^{87} N_{1}^{436} N_{2}^{232} N_{3}^{67}} \\
& +\sqrt[256]{G^{81} N_{1}^{364} N_{2}^{152} N_{3}^{69}}+\sqrt[64]{G^{21} N_{1}^{92} N_{2}^{32} N_{3}^{21}} \\
& +\sqrt[512]{G^{171} N_{1}^{740} N_{2}^{248} N_{3}^{183}}+\sqrt[64]{G^{21} N_{1}^{92} N_{2}^{28} N_{3}^{21}} \\
& +\sqrt[256]{G^{87} N_{1}^{372} N_{2}^{104} N_{3}^{99}} \\
& +\left(H x\left(N_{1} N_{2} N_{3}\right)^{-1}\right)^{1 / 2}+x^{\theta} \\
& \ll \sqrt[48]{x^{15} N_{1}^{38} N_{2}^{-6} N_{3}^{4}}+\sqrt[64]{x^{15} N_{1}^{54} N_{2}^{10} N_{3}^{12}} \\
& +\sqrt[32]{x^{9} N_{1}^{26} N_{2}^{-6} N_{3}^{8}}+\sqrt[320]{x^{87} N_{1}^{262} N_{2}^{58} N_{3}^{-20}} \\
& +\sqrt[256]{x^{81} N_{1}^{202} N_{2}^{-10} N_{3}^{-12}}+\sqrt[64]{x^{21} N_{1}^{50} N_{2}^{-10}} \\
& +\sqrt[512]{x^{171} N_{1}^{398} N_{2}^{-94} N_{3}^{12}}+\sqrt[64]{x^{21} N_{1}^{50} N_{2}^{-14}} \\
& +\sqrt[256]{x^{87} N_{1}^{198} N_{2}^{-70} N_{3}^{12}} \\
& +\left(H x\left(N_{1} N_{2} N_{3}\right)^{-1}\right)^{1 / 2}+x^{\theta} \\
& \ll \sqrt[48]{x^{17} N_{1}^{34} N_{2}^{-10}}+\sqrt[64]{x^{21} N_{1}^{42} N_{2}^{-2}} \\
& +\sqrt[32]{x^{13} N_{1}^{18} N_{2}^{-14}}+\sqrt[320]{x^{87} N_{1}^{262} N_{2}^{38}} \\
& +\sqrt[256]{x^{81} N_{1}^{202} N_{2}^{-22}}+\sqrt[64]{x^{21} N_{1}^{50} N_{2}^{-10}} \\
& +\sqrt[512]{x^{177} N_{1}^{386} N_{2}^{-106}}+\sqrt[256]{x^{93} N_{1}^{186} N_{2}^{-82}} \\
& +\left(H x\left(N_{1} N_{2} N_{3}\right)^{-1}\right)^{1 / 2}+x^{\theta} .
\end{aligned}
$$

From (2.30) and (2.31) we deduce, provided that (2.28) is false, that

$$
x^{-3 \eta} \Phi(H ; \boldsymbol{N}) \ll \sum_{1 \leq i \leq 8} B_{i}+\left(H x\left(N_{1} N_{2} N_{3}\right)^{-1}\right)^{1 / 2}+x^{\varphi},
$$

where

$$
\begin{aligned}
B_{1} & =\min \left(\sqrt[4]{x N_{1} N_{2}^{2}}, \sqrt[48]{x^{17} N_{1}^{34} N_{2}^{-10}}\right) \\
& \leq\left(x^{61} J^{78}\right)^{1 / 224} \ll x^{0.364}, \\
B_{2} & =\min \left(\sqrt[4]{x N_{1} N_{2}^{2}}, \sqrt[64]{x^{21} N_{1}^{42} N_{2}^{-2}}\right) \\
& \leq\left(x^{65} J^{86}\right)^{1 / 240} \ll x^{0.365}, \\
B_{3} & =\min \left(\sqrt[4]{x N_{1} N_{2}^{2}}, \sqrt[32]{x^{13} N_{1}^{18} N_{2}^{-14}}\right) \\
& \leq\left(x^{45} J^{50}\right)^{1 / 160} \ll x^{0.364}, \\
B_{4} & =\min \left(\sqrt[4]{x N_{1} N_{2}^{2}}, \sqrt[256]{x^{81} N_{1}^{202} N_{2}^{-22}}\right)
\end{aligned}
$$




$$
\begin{aligned}
& \leq\left(x^{305} J^{426}\right)^{1 / 1152} \ll x^{0.363}, \\
B_{5} & =\min \left(\sqrt[4]{x N_{1} N_{2}^{2}}, \sqrt[320]{x^{87} N_{1}^{262} N_{2}^{38}}\right) \\
& \leq\left(x^{311} J^{486}\right)^{1 / 1216} \ll x^{0.361}, \\
B_{6} & =\min \left(\sqrt[4]{x N_{1} N_{2}^{2}}, \sqrt[64]{x^{21} N_{1}^{50} N_{2}^{-10}}\right) \\
& \leq\left(x^{81} J^{110}\right)^{1 / 304} \ll x^{0.362}, \\
B_{7} & =\min \left(\sqrt[4]{x N_{1} N_{2}^{2}}, \sqrt[512]{x^{177} N_{1}^{386} N_{2}^{-106}}\right) \\
& \leq\left(x^{669} J^{878}\right)^{1 / 2480} \ll x^{0.363}, \\
B_{8} & =\min \left(\sqrt[4]{x N_{1} N_{2}^{2}}, \sqrt[256]{x^{93} N_{1}^{186} N_{2}^{-82}}\right) \\
& \leq\left(x^{361} J^{454}\right)^{1 / 1328} \ll x^{0.362} .
\end{aligned}
$$

From (2.29), (2.32) to (2.40), we always have

$$
x^{-3 \eta} \Phi(H ; \boldsymbol{N}) \ll E_{4}(H)+x^{\varphi} .
$$

We put (2.41) in (1) of [3] and choose $K$ optimally to get

$$
\begin{aligned}
x^{-4 \eta} S(2,2,1,1 ; \boldsymbol{N}) \ll & \sqrt[42]{G^{15}\left(N_{1} N_{2}\right)^{31} N_{3}^{15}} \\
& +\sqrt[13]{G^{5} N_{1}^{9} N_{2}^{9} N_{3}^{5}}+\sqrt[54]{G^{15}\left(N_{1} N_{2}\right)^{47} N_{3}^{15}} \\
& +\sqrt[16]{G^{5}\left(N_{1} N_{2}\right)^{13} N_{3}^{5}}+\sqrt[7]{G^{2} N_{1}^{6} N_{2}^{6} N_{3}^{2}} \\
& +\sqrt[29]{G^{10}\left(N_{1} N_{2}\right)^{22} N_{3}^{10}}+\sqrt[5]{G^{2} N_{1}^{3} N_{2}^{3} N_{3}^{2}} \\
& +\sqrt[20]{\left(N_{1} N_{2}\right)^{14} N_{3}^{13}}+\sqrt[20]{\left(N_{1} N_{2}\right)^{16} N_{3}^{7}} \\
& +\sqrt[18]{G^{5}\left(N_{1} N_{2}\right)^{14} N_{3}^{5}}+x^{\varphi} \\
\ll & \sqrt[42]{x^{15} J}+\sqrt[13]{x^{5} J^{-1}}+\sqrt[54]{x^{15} J^{17}} \\
& +\sqrt[16]{x^{5} J^{3}}+\sqrt[7]{x^{2} J^{2}}+\sqrt[29]{x^{10} J^{-1}} \\
& +\sqrt[5]{x^{2} J^{-1}}+\sqrt[20]{x^{6.5} J}+\sqrt[20]{x^{3.5} J^{9}} \\
& +\sqrt[18]{x^{5} J^{4}}+x^{\varphi} \\
\ll & \sqrt[13]{x^{5} J^{-1}}+\sqrt[5]{x^{2} J^{-1}}+x^{\varphi}
\end{aligned}
$$

Now the required estimate follows from (2.42) if $J \geq x^{4 / 19}$, and otherwise it is a consequence of (2.27).

3. Proof of Theorem 3. The underlying idea is the same as used in proving Theorem 2, but the details are now much simpler, because we are dealing with exponential sums of a lower dimension. We use conventions introduced in Section 2 of [4]. We consider the sum $S_{a, b, c}(M, N ; x)$, where $(a, b, c)$ is a permutation of $(1,1,2)$. If $(a, b, c)=(1,1,2)$, then similarly to (2.9) we have

(3.1) $x^{-3 \eta} \Phi(H, M, N) \ll(H G)^{13 / 16}+\sqrt[32]{H^{19} G^{22} N_{2}^{4} N_{3}^{4}}+\sqrt[4]{G^{4} H^{3}}+x^{1 / 3}$. 
In fact, we can produce a new variable $w=u v$ from (4) of [4]. The following arguments are exactly those stated from (2.7) to (2.9), the only difference is that we now have " $n_{1}=1$ " in those expressions. We put the estimate (3.1) in (1) of [4] and choose $K$ optimally to get

$$
\begin{aligned}
x^{-4 \eta} S_{1,1,2}(M, N ; x) & \ll \sqrt[58]{x^{13}(M N)^{13}}+\sqrt[51]{x^{11}(M N)^{12}}+\sqrt[7]{x^{2} M N}+x^{1 / 3} \\
& \ll x^{5 / 14} .
\end{aligned}
$$

For $(a, b, c)=(2,1,1)$, by $(3)$ of $[4]$ we have

$$
\begin{aligned}
\Phi(H, M, N) \ll & H^{-1}\left(M^{2}(H G)^{-1}\right)^{1 / 2} \sum_{h \sim H} \sum_{u \cong U} \mid \sum_{n \in I} Q(n) e\left(C\left(\text { xhun }^{-2}\right)^{1 / 2}\right) \mid \\
& +(H G)^{1 / 2}+x^{1 / 3},
\end{aligned}
$$

where $I$ denotes an interval contained in $[N, 2 N]$, and $U=H G M^{-1}$. We use Lemma 1.6 of [2] to relax the range of $n$, and get

$$
\begin{aligned}
& x^{-\eta} \Phi(H, M, N) \\
& \ll H^{-1}\left(M^{2}(H G)^{-1}\right)^{1 / 2} \sum_{h \sim H} \sum_{u \cong U} \mid \sum_{n \sim N} Q(n) e\left(C\left(\text { xhun }^{-2}\right)^{1 / 2}+n t\right) \mid \\
& \quad+(H G)^{1 / 2}+x^{1 / 3},
\end{aligned}
$$

where $t$ is a real number, $t \in[0,1)$, and it is independent of the other variables. We produce a new variable $r=h u$ from (3.2) and get

$$
\begin{aligned}
& x^{-2 \eta} \Phi(H, M, N) \\
& \ll H^{-1}\left(M^{2}(H G)^{-1}\right)^{1 / 2} \sum_{r \cong R}\left|\sum_{n \sim N} Q(n) e\left(C\left(x r n^{-2}\right)^{1 / 2}+n t\right)\right| \\
& \quad+(H G)^{1 / 2}+x^{1 / 3},
\end{aligned}
$$

where $R=H^{2} G M^{-1}$. We apply Lemma 1.1 to the triple sum in (3.2) with $(h, x, y)=(h, u, n)$ to obtain

$$
\begin{aligned}
x^{-2 \eta} \Phi(H, M, N) \ll & \sqrt[22]{H^{8} G^{11} N^{13} M^{3}}+(H G)^{1 / 2} N^{5 / 8} \\
+ & \sqrt[16]{(H G)^{4} N^{17}}+\sqrt[32]{H^{8} G^{11} N^{28} M^{3}} \\
& +\sqrt[32]{H^{13} G^{16} N^{18} M^{3}}+\sqrt[4]{G N^{4} M} \\
& +\sqrt[4]{H G^{2} N^{2} M}+\left(H x(M N)^{-1}\right)^{1 / 2}+x^{1 / 3} \\
=: & E_{5}(H)+\sqrt[22]{H^{8} G^{11} N^{13} M^{3}} \\
& +\sqrt[4]{H G^{2} N^{2} M}, \text { say. }
\end{aligned}
$$

If $H \leq M$, then Lemma 1.1 is applicable to the exponential sum of (3.3) 
with $(h, x, y)=(1, n, r)$, and we get

$$
\begin{aligned}
x^{-3 \eta} \Phi(H, M, N) \ll & \sqrt[22]{H^{-4} G^{5} N^{19} M^{9}}+\sqrt[8]{H^{-2} G N^{8} M^{3}} \\
& +\sqrt[16]{H^{6} G^{5} N^{16} M^{-1}}+\sqrt[32]{H^{6} G^{10} N^{29} M^{4}} \\
& +\sqrt[32]{H^{-9} G^{5} N^{29} M^{14}}+\sqrt[4]{H^{2} G^{2} N^{3}} \\
& +\sqrt[4]{H^{-1} G N^{3} M^{2}}+\left(H x(M N)^{-1}\right)^{1 / 2}+x^{1 / 3} \\
=: & E_{6}(H), \text { say. }
\end{aligned}
$$

If $H>M$, then from (3.4) we get

$$
x^{-2 \eta} \Phi(H, M, N) \ll E_{5}(H)+\sqrt[22]{H^{11} G^{11} N^{13}}+(H G N)^{1 / 2} .
$$

By (3.5) and (3.6) we always have

$$
x^{-3 \eta} \Phi(H, M, N) \ll E_{5}(H)+E_{6}(H)+\sqrt[22]{H^{11} G^{11} N^{13}} .
$$

From (3.4) and (3.7) we deduce that

$$
x^{-3 \eta} \Phi(H, M, N) \ll E_{5}(H)+\sqrt[22]{H^{11} G^{11} N^{13}}+R_{1}+R_{2},
$$

where

$$
R_{1}=\min \left(E_{6}(H), \sqrt[22]{H^{8} G^{11} N^{13} M^{3}}\right) \ll E_{7}(H)+\sum_{1 \leq i \leq 4} D_{i},
$$

$$
\begin{aligned}
E_{7}(H)= & \sqrt[16]{H^{6} G^{5} N^{16} M^{-1}}+\sqrt[32]{H^{6} G^{10} N^{29} M^{4}} \\
& +\sqrt[4]{H^{2} G^{2} N^{3}}+\left(H x(M N)^{-1}\right)^{1 / 2}+x^{1 / 3} \\
D_{1}= & \min \left(\sqrt[22]{H^{-4} G^{5} N^{19} M^{9}}, \sqrt[22]{H^{8} G^{11} N^{13} M^{3}}\right) \\
\ll & \sqrt[66]{G^{21} M^{21} N^{51}} \ll \sqrt[66]{x^{21} N^{9}}, \\
D_{2}= & \min \left(\sqrt[8]{H^{-2} G N^{8} M^{3}}, \sqrt[22]{H^{8} G^{11} N^{13} M^{3}}\right) \\
\ll & \sqrt[54]{G^{15} N^{45} M^{15}} \ll \sqrt[54]{x^{15} N^{15}} \\
D_{3}= & \min \left(\sqrt[32]{H^{-9} G^{5} N^{29} M^{14}}, \sqrt[22]{H^{8} G^{11} N^{13} M^{3}}\right) \\
\ll & \sqrt[454]{G^{139} N^{349} M^{139}} \ll \sqrt[454]{x^{139} N^{71}}, \\
D_{4}= & \min \left(\sqrt[4]{H^{-1} G N^{3} M^{2}}, \sqrt[22]{H^{8} G^{11} N^{13} M^{3}}\right) \\
\ll & \sqrt[54]{G^{19} N^{37} M^{19}} \ll x^{19 / 54}
\end{aligned}
$$

moreover,

$$
R_{2}=\min \left(E_{6}(H), \sqrt[4]{H G^{2} N^{2} M}\right) \ll E_{7}(H)+\sum_{5 \leq i \leq 8} D_{i},
$$

where 


$$
\begin{aligned}
D_{5} & =\min \left(\sqrt[22]{H^{-4} G^{5} N^{19} M^{9}}, \sqrt[4]{H G^{2} N^{2} M}\right) \\
& \ll \sqrt[38]{G^{13} N^{27} M^{13}} \ll \sqrt[38]{x^{13} N}, \\
D_{6} & =\min \left(\sqrt[8]{H^{-2} G N^{8} M^{3}}, \sqrt[4]{H G^{2} N^{2} M}\right) \ll \sqrt[16]{G^{5} N^{12} M^{5}} \\
& \ll \sqrt[16]{x^{5} N^{2}} \\
D_{7} & =\min \left(\sqrt[32]{H^{-9} G^{5} N^{29} M^{14}}, \sqrt[4]{H G^{2} N^{2} M}\right) \\
& \ll \sqrt[68]{G^{23} N^{47} M^{23}} \ll \sqrt[68]{x^{23} N}, \\
D_{8} & =\min \left(\sqrt[4]{H^{-1} G N^{3} M^{2}}, \sqrt[4]{H G^{2} N^{2} M}\right) \ll \sqrt[8]{G^{3} N^{5} M^{3}} \\
& \ll \sqrt[8]{x^{3} N^{-1}}
\end{aligned}
$$

As $N \ll x^{1 / 4}$, we see that $D_{i} \ll x^{0.353}$ for $1 \leq i \leq 7$. By (3.8) to (3.19) we get

$$
\begin{aligned}
x^{-3 \eta} \Phi(H, M, N) \ll & E_{5}(H)+E_{7}(H)+\sqrt[8]{x^{3} N^{-1}} \\
& +\sqrt[22]{H^{11} G^{11} N^{13}}+x^{\psi}
\end{aligned}
$$

$\psi=29 / 80$. We put the estimate of (3.20) in (1) of [4] and choose an optimal $K$ to get

$$
\begin{aligned}
x^{-4 \eta} S_{2,1,1}(M, N ; x) \ll & \sqrt[20]{G^{4} N^{21} M^{4}}+\sqrt[12]{G^{4} N^{9} M^{4}} \\
& +\sqrt[40]{G^{11} N^{36} M^{11}}+\sqrt[45]{G^{16} N^{31} M^{16}} \\
& +\sqrt[4]{x N^{2}}+\sqrt[22]{G^{5} N^{22} M^{5}} \\
& +\sqrt[38]{G^{10} N^{35} M^{10}}+\sqrt[6]{G^{2} N^{5} M^{2}} \\
& +\sqrt[33]{G^{11} M^{11} N^{24}}+\sqrt[8]{x^{3} N^{-1}}+x^{\psi} \\
\ll & \sqrt[40]{x^{11} N^{14}}+\sqrt[4]{x N^{2}} \\
& +\sqrt[6]{x^{2} N}+\sqrt[8]{x^{3} N^{-1}} \\
& +\sqrt[22]{x^{5} N^{12}}+\sqrt[38]{x^{10} N^{15}}+x^{\psi}
\end{aligned}
$$

We remove the smooth coefficient $Q(n)$ in (3.3) by a partial summation, and we then relax the summation range for $n$ by means of Lemma 1.6 of [2]. This yields

$$
\begin{aligned}
& x^{-3 \eta} \Phi(H, M, N) \\
& \ll H^{-1}\left(M^{2}(H G)^{-1}\right)^{1 / 2} \sum_{r \cong R}\left|\sum_{n \sim N} e\left(C\left(x r n^{-2}\right)^{1 / 2}+\xi n\right)\right| \\
& \quad+(H G)^{1 / 2}+x^{1 / 3} \\
& =: H^{-1} M(H G)^{-1 / 2} S+(H G)^{1 / 2}+x^{1 / 3}, \quad \text { say, }
\end{aligned}
$$

where $\xi$ is some real number, $0 \leq \xi<1$, independent of $r$ and $n$. Let 
$Q \in\left(100, N x^{-\delta}\right)$ be a number to be chosen later $\left(\delta=\varepsilon^{2}\right)$. By Cauchy's inequality and Weyl's inequality (Lemma 1.3 of [2]),

$$
x^{-\eta} S^{2} \ll(R N)^{2} Q^{-1}+R^{3 / 2} N Q^{-1}\left|\sum_{(n, q) \in D} \sum_{r \cong R} r^{-1 / 2} e(f(n, q, r))\right|,
$$

where, for some $Q_{1} \in[1, Q], D=\left\{(n, q) \mid q \sim Q_{1}, n, n+q \sim N\right\}$, and $f(n, q, r)=C\left(x r^{-1}\right)^{1 / 2}\left((n+q)^{-1}-n^{-1}\right)+q \xi$. We can use Lemma 1.4 of [2] to transform the summation over $r$, and we get a summation over $w \cong M Q_{1}(\mathrm{NH})^{-1}$. We then exchange the order of summation and estimate the sum over $w$ trivially to obtain, with some $w$, the estimate

$$
\begin{aligned}
& R^{3 / 2} N Q^{-1}\left|\sum_{(n, q) \in D} \sum_{r \cong R} r^{-1 / 2} e(f(n, q, r))\right| \\
& \ll \sqrt{H^{5} G^{3} M^{-2} N Q^{-1}}\left|\sum_{(n, q) \in D} e(F(n, q))\right| \\
& \quad+\sqrt{Q^{-1} H^{7} N^{5} M^{-4} G^{3}}+H^{3} G M^{-2} N^{3} Q^{-1}+G H^{2} M^{-1} N^{2} \ln x,
\end{aligned}
$$

where $F(n, q)=C^{\prime}(x w)^{1 / 3}\left((n+q)^{-1}-n^{-1}\right)^{2 / 3}+\xi q$. It is easy to verify that

$$
F(n, q) \underset{\Delta}{\sim} C^{\prime}(x w)^{1 / 3} n^{-4 / 3} q^{2 / 3}, \quad \Delta=Q_{1} N^{-1} .
$$

Thus Lemma 1.5 of [2] yields

$$
\begin{aligned}
x^{-\eta} & \sqrt{H^{5} G^{3} Q^{-1} M^{-2} N}\left|\sum_{(n, q) \in D} e(F(n, q))\right| \\
\ll & \sqrt[6]{H^{17} G^{11} Q^{2} N^{4} M^{-6}}+\sqrt[6]{H^{15} G^{9} Q^{2} M^{-6} N^{8}} \\
& +\sqrt[10]{H^{27} G^{17} Q^{10} M^{-10} N^{4}}+\sqrt[8]{H^{19} G^{11} Q^{3} M^{-8} N^{12}} \\
& +\sqrt[4]{H^{9} G^{5} Q M^{-4} N^{7}}+\sqrt[4]{H^{10} G^{6} Q^{3} M^{-4} N^{4}}+\sqrt{H^{5} G^{3} M^{-2} N^{3}} \\
= & L_{1}(Q) .
\end{aligned}
$$

From (3.23) to (3.25) we get

$$
x^{-2 \eta} S^{2} \ll(R N)^{2} Q^{-1}+\sqrt{Q^{-1} H^{7} N^{5} M^{-4} G^{3}}+L_{1}(Q)=: L_{2}(Q) .
$$

Obviously (3.26) also holds if $Q \ll 1$. By Lemma 3 of [3], there is a $Q \in$ $\left(0, N x^{-\delta}\right)$ such that

$$
\begin{aligned}
x^{-\eta} L_{2}(Q) \ll & \sqrt[8]{H^{25} G^{15} M^{-10} N^{8}}+\sqrt[8]{H^{23} G^{13} N^{12} M^{-10}} \\
& +\sqrt[20]{H^{67} G^{37} N^{24} M^{-30}}+\sqrt[11]{H^{31} G^{17} N^{18} M^{-14}} \\
& +\sqrt[5]{H^{13} G^{7} N^{9} M^{-6}}+\sqrt[7]{H^{22} G^{12} N^{10} M^{-10}} \\
& +\left(H^{5} G^{3} M^{-2} N^{3}\right)^{1 / 2}+\sqrt[10]{H^{31} G^{17} N^{14} M^{-14}}
\end{aligned}
$$




$$
\begin{aligned}
& +\sqrt[10]{H^{29} G^{15} N^{18} M^{-14}}+\sqrt[30]{H^{97} G^{47} M^{-50} N^{54}} \\
& +\sqrt[14]{H^{40} G^{20} M^{-20} N^{27}}+\sqrt[3]{H^{8} G^{4} M^{-4} N^{6}} \\
& +\sqrt[10]{H^{31} G^{15} M^{-16} N^{19}}+H^{4} G^{2} M^{-2} N \\
& +\sqrt{H^{7} N^{4} M^{-4} G^{3}}
\end{aligned}
$$

From (3.22), (3.26) and (3.27) we have

$$
x^{-6 \eta} \Phi(H, M, N) \ll L_{+}(H)+L_{-}(H)+x^{0.36},
$$

where

$$
\begin{aligned}
L_{+}(H)= & \sqrt[16]{H G^{7} M^{6} N^{8}}+\sqrt[40]{H^{7} G^{17} N^{24} M^{10}} \\
& +\sqrt[14]{H G^{5} N^{10} M^{4}}+\sqrt[20]{H G^{7} N^{14} M^{6}} \\
& +\sqrt[60]{H^{7} G^{17} M^{10} N^{54}}+\sqrt[20]{H G^{5} M^{4} N^{19}} \\
& +(H G N)^{1 / 2}+\sqrt[4]{H N^{4} G} \\
L_{-}(H)= & \sqrt[16]{H^{-1} G^{5} M^{6} N^{12}}+\sqrt[22]{H^{-1} G^{6} N^{18} M^{8}} \\
& +\sqrt[10]{H^{-2} G^{2} N^{9} M^{4}} \\
& +\sqrt[4]{H^{-1} G N^{3} M^{2}}+\sqrt[20]{H^{-1} G^{5} M^{6} N^{18}} \\
& +\sqrt[28]{H^{-2} G^{6} N^{27} M^{8}}+\sqrt[6]{H^{-1} G M^{2} N^{6}}
\end{aligned}
$$

If $H \geq \sqrt[13]{G^{-7} M^{5} N^{8}}$, we have

$$
\begin{aligned}
L_{-}(H) \ll & \sqrt[286]{G^{85} N^{226} M^{99}}+\sqrt[130]{G^{40} N^{101} M^{42}} \\
& +\sqrt[260]{G^{72} M^{73} N^{226}}+\sqrt[364]{G^{92} M^{98} N^{335}} \\
& +\sqrt[78]{G^{20} M^{21} N^{70}}+\sqrt[52]{G^{20} M^{21} N^{31}}+x^{0.36} \\
\ll & \sqrt[286]{x^{85} N^{56} M^{14}}+\sqrt[130]{x^{40} N^{21} M^{2}} \\
& +\sqrt[260]{x^{72} M N^{82}}+\sqrt[364]{x^{92} M^{6} N^{151}} \\
& +\sqrt[78]{x^{20} M N^{30}}+\sqrt[52]{x^{20} M N^{-9}}+x^{0.36} \\
\ll & \sqrt[52]{x^{20} M N^{-9}}+x^{0.361}
\end{aligned}
$$

because $G=x\left(M N^{2}\right)^{-1}, M \gg N$ and $M N \ll x^{1 / 2}$. If $H<\sqrt[13]{G^{-7} M^{5} N^{8}}$, by (11) of [4] we know that

$$
\begin{aligned}
x^{-2 \eta} \Phi(H, M, N) \ll & \sqrt[52]{G^{20} M^{21} N^{31}}+\sqrt[208]{G^{60} M^{89} N^{145}} \\
& +\sqrt[1040]{G^{348} M^{225} N^{841}}+\sqrt[832]{G^{324} M^{231} N^{575}} \\
& +\sqrt[208]{G^{84} M^{70} N^{125}}+\sqrt[832]{G^{348} M^{329} N^{425}} \\
& +\sqrt[4]{(H G)^{3} M}+\sqrt[128]{(H G)^{114} M^{3} N^{5}}+x^{1 / 3} \\
\ll & \sqrt[52]{x^{20} M N^{-9}}+\sqrt[208]{x^{60} M^{29} N^{25}} \\
& +\sqrt[1040]{x^{348} M^{-123} N^{145}}+\sqrt[832]{x^{324} M^{-93} N^{-73}}
\end{aligned}
$$




$$
\begin{aligned}
& +\sqrt[208]{x^{84} M^{-14} N^{-43}}+\sqrt[832]{x^{348} M^{-19} N^{-271}} \\
& +\sqrt[4]{(H G)^{3} M}+\sqrt[128]{(H G)^{114} M^{3} N^{5}}+x^{1 / 3} \\
\ll & \sqrt[52]{x^{20} M N^{-9}}+\sqrt[832]{x^{324} N^{-166}} \\
& +\sqrt[208]{x^{84} N^{-57}}+\sqrt[832]{x^{348} N^{-290}} \\
& +x^{\psi}+\sqrt[4]{(H G)^{3} M}+\sqrt[128]{(H G)^{114} M^{3} N^{5}} \\
= & P_{1}+\sqrt[4]{(H G)^{3} M}+\sqrt[128]{(H G)^{114} M^{3} N^{5}}
\end{aligned}
$$

From (3.28) to (3.32) we always have

(3.33) $x^{-6 \eta} \Phi(H, M, N) \ll L_{+}(H)+P_{1}+\sqrt[4]{(H G)^{3} M}+\sqrt[128]{(H G)^{114} M^{3} N^{5}}$.

If $H>\left(G^{-2} M N^{3}\right)^{1 / 4}$, then similarly to (3.31) we easily verify that

$$
L_{-}(H) \ll \sqrt[16]{x^{6} M N^{-3}}+x^{\psi}
$$

thus from (3.28) and (3.34) we have

$$
x^{-6 \eta} \Phi(H, M, N) \ll L_{+}(H)+\sqrt[16]{x^{6} M N^{-3}}+x^{\psi} .
$$

If $H \leq\left(G^{-2} M N^{3}\right)^{1 / 4}$, then from (3.33) we get

(3.36) $x^{-6 \eta} \Phi(H, M, N) \ll L_{+}(H)+P_{1}+\sqrt[16]{x^{6} M N^{-3}}+\sqrt[128]{(H G)^{114} M^{3} N^{5}}$.

From (3.35) and (3.36) we always have

(3.37) $x^{-6 \eta} \Phi(H, M, N) \ll L_{+}(H)+P_{1}+\sqrt[16]{x^{6} M N^{-3}}+\sqrt[128]{(H G)^{114} M^{3} N^{5}}$.

If $H<\left(G^{-82} M^{61} N^{91}\right)^{1 / 146}$, by (3.37) we see readily that

$$
\begin{aligned}
x^{-6 \eta} \Phi(H, M, N) \ll & L_{+}(H)+P_{1}+\sqrt[16]{x^{6} M N^{-3}} \\
& +\sqrt[584]{x^{228} M^{3} N^{-109}} \\
= & : L_{+}(H)+P_{2} .
\end{aligned}
$$

If $H \geq\left(G^{-82} M^{61} N^{91}\right)^{1 / 146}$ then similarly to (3.31) we verify that

$$
L_{-}(H) \ll \sqrt[584]{x^{228} M^{3} N^{-109}}+x^{\psi} .
$$

From (3.28) and (3.39) we find that (3.38) is always true. We now put the estimate of (3.38) in (1) of [4] and then choose $K$ optimally via Lemma 3 of [3] to infer that

$$
x^{-7 \eta} S_{2,1,1}(M, N ; x) \ll P_{2}+\sqrt[17]{x^{7} N^{-5}}+\sqrt[47]{x^{17} N^{-3}}=: P_{3}
$$

If $N \geq x^{7 / 40}$ from (3.40) we have

$$
x^{-7 \eta} S_{2,1,1}(M, N ; x) \ll P_{3} \ll x^{\psi}
$$

(for instance, $\sqrt[16]{x^{6} M N^{-3}} \ll \sqrt[160]{x^{53}(M N)^{10}} \ll x^{\psi}$ ), and if $N<x^{7 / 40}$, by (3.21) it is easy to see that

$$
x^{-4 \eta} S_{2,1,1}(M, N ; x) \ll \sqrt[8]{x^{3} N^{-1}}+x^{\psi},
$$


and by (8) of [4] we also have

$$
x^{-\eta} S_{2,1,1}(M, N ; x) \ll\left(x^{23} N^{27}\right)^{1 / 73},
$$

thus

$$
x^{-4 \eta} S_{2,1,1}(M, N ; x) \ll \min \left(\sqrt[8]{x^{3} N^{-1}}, \sqrt[73]{x^{23} N^{27}}\right)+x^{\psi} \ll x^{\psi},
$$

insofar as the desired result for the case $(a, b, c)=(2,1,1)$ also holds. (The bound given in [5], worse than (3.41), suffices here yet.)

Similarly and more easily, we can show that

$$
x^{-7 \eta} S_{1,2,1}(M, N ; x) \ll x^{\psi} .
$$

This finishes the proof of Theorem 3.

\section{References}

[1] H.-Q. Liu, On the number of abelian groups of a given order (supplement), Acta Arith. 64 (1993), 285-296.

[2] - , The greatest prime factor of the integers in an interval, ibid. 65 (1993), 301-328.

[3] - , The distribution of 4-full numbers, ibid. 67 (1994), 165-176.

[4] —, On some divisor problems, ibid. 68 (1994), 193-200.

[5] —, The number of squarefull numbers in an interval, ibid. 64 (1993), 129-149.

206-10, BAO GUO STREET

HARBIN, 150066

CHINA 